(Submitted to Numerical Heat Transfer A: Applications)

\title{
Magnetic Damping of g-Jitter Induced Double-Diffusive Convection
}

\author{
Y. Shu and B. Q. $\mathrm{Li}^{*}$ \\ Department of Mechanical Engineering \\ Washington State University \\ Pullman, LA 70803 \\ Email:1i@mme.wsu.edu \\ and \\ H. C. de Groh \\ NASA Lewis Research Center \\ Cleveland, $\mathrm{OH} 44135$
}

August, 2001

This report is a preprint of an article submitted to a journal for publication. Because of changes that may be made before formal publication, this preprint is made available with the understanding that it will not be cited or reproduced without the permission of the author.

- To whom correspondence should be made. 


\begin{abstract}
This paper describes a numerical study of the g-jitter driven double diffusive convective flows, thermal and concentration distributions in binary alloy melt systems subject to an external magnetic field. The study is based on the finite element solution of transient magnetohydrodynamic equations governing the momentum, thermal and solutal transport in the melt pool. Numerical simulations are conducted using the synthesized single- and multifrequency g-jitter as well as the real g-jitter data taken during space flights with or without an applied magnetic field. It is found that for the conditions studied, the main melt flow follows approximately a linear superposition of velocity components induced by individual g-jitter components, regardless of whether a magnetic field exists or not. The flow field is characterized by a recirculating double diffusive convection loop oscillating in time with a defined frequency equal to that of the driving g-jitter force. An applied magnetic field has little effect on the oscillating recirculating pattern, except around the moment in time when the flow reverses its direction. The field has no effect on the oscillation period, but it changes the phase angle. It is very effective in suppressing the flow intensity and produces a notable reduction of the solutal striation and time fluctuations in the melt. For a given magnetic field strength, the magnetic damping effect is more pronounced on the velocity associated with the largest g-jitter component present and/or the g-jitter spiking peaks. A stronger magnetic field is more effective in suppressing the melt convection and also is more helpful in bringing the convection in phase with the g-jitter driving force. The applied field is particularly useful in suppressing the effect of real g-jitter spikes on both flow and solutal distributions. With appropriately selected magnetic fields, the convective flows caused by g-jitter can be reduced sufficiently and diffusion dominant. solutal transport in the melt is possible.
\end{abstract}




\section{NOMENCLATURE}

b, B applied magnetic field, its magnitude

$C_{P} \quad$ heat capacity

$C_{H} C_{L} \quad$ maximum and minimum concentration

$\triangle C \quad$ concentration difference

$C_{0}$

$\mathrm{e}$

dimensionless concentration

unit vector

$f$

dimensionless frequency of the acceleration

$f^{*}$

frequency of the acceleration

$g$

dimensionless amplitude of the acceleration

$g^{*}$

amplitude of the acceleration

$g_{0}$

earth gravity

$\mathrm{Ha}$

Hartmann number, $H a=B L \sqrt{\sigma / \mu}$

$G r_{s}$

solutal Grashof number, $G r_{S}=g \beta_{C} \Delta C L^{3} / v^{2}$

$G r_{T}$

$\hat{i}, \hat{j}$

thermal Grashof number, $G r_{T}=g \beta_{T} \Delta T L^{3} / v^{2}$

unit vectors of the ith, $\mathrm{jth}$ components

thermal conductivity

$k$

$L$

$\mathbf{P}$

length of the square cavity

discretized nodal pressure array

Pr Prandil number. $\operatorname{Pr}=\frac{v}{\alpha}$

$S c$

$T$

schmidt number, $S c=\frac{v}{D}$

dimensionless temperature

$T_{H}, T_{L}$

maximum and minimum temperatures

$\Delta T$

u

temperature between $T_{H}$ and $T_{L}$

$\mathrm{u}, \mathrm{v}$

$V$

dimensionless velocity

$\mathrm{x}$ and $\mathrm{y}$ component of the dimensionless velocity

volume of the cavity

$x, y$

dimensionless coordinates

\section{Greek}

$\alpha$

thermal diffusivity

$\beta_{C} \quad$ solutal expansion coefficient

$\beta_{T} \quad$ thermal expansion coefficient

$D \quad$ solutal diffusivity

$\phi$

$\theta$

shape function for velocity

shape function for temperature and concentration

shape function for pressusre 


$\begin{array}{ll}\mu & \text { dynamic viscosity } \\ v & \text { kinematic viscosity } \\ \partial \Omega & \text { boundary of computational domain } \\ \nabla & \text { gradient operator } \\ \rho & \text { density } \\ \Omega & \text { computational domain }\end{array}$

\section{Subscripts}

$\begin{array}{ll}i, j & \text { the } i \text { th }, j \text { th point } \\ n & \text { the nth component }\end{array}$

\section{Superscripts}
$i, j$
the ith, jth component
$T$
matrix transpose 


\section{INTRODUCTION}

During space flights, perturbation from idealized dynamic weightlessness conditions established in Space Shuttles arises from a variety of disturbances such as crew motions, mechanical vibrations (pumps, motors, excitations of natural frequencies of spacecraft structures), spacecraft maneuvers and attitude, atmosphere drag and the Earth's gravity gradient [1]. Some typical signatures of gravity perturbation or more often referred to as g-jitter, taken by astronauts using on-board accelerometer during a typical Space Shuttle flight, are illustrated in Figure $1 \mathrm{~A}$ Although small in magnitude, $\mathrm{g}$-jitter is random in both direction and time, and causes time dependent convection in melt being processed in space. Experiments on melt growth of crystals conducted in Space Shuttles suggest that the g-jitter induced melt flow has deleterious effects on the solute uniformity and defect formation in the space grown crystals and is the main cause for the non-repeatability of the quality processed in microgravity [2,3].

Direct study of the g-jitter effects on the thermal and fluid flow behavior of melt growth systems in space environment is difficult because of limited instrumentation precision. Numerical simulations useful in providing a basic understanding of the physics governing the g-jitter induced phenomena. Consequently, both 2D and 3D numerical models have been developed and applied to estimate the effects associated with both idealized singleand multiple-frequency g-jitter modulations and realistic g-jitter data collected by accelerometer during actual flight experiments. Calculations suggested that the frequency, amplitude and spatial orientation of the residual gravity vector all play an important role in determining the convective flow behavior of the system. The most detrimental effects occur when g-jitter is perpendicular to the applied thermal gradient [4-7]. For solidification or crystal growth applications, space experiments and numerical simulations both indicate that $\mathrm{g}$-jitter components with lower frequencies are most harmful and are repsponsible for defects and solutal striation in the crystals produced in Space Shuttles.

The need to have a quiescent melt pool from which crystals are grown has motivated researchers to develop and apply various concepts to suppress the deleterious g-jitter induced convection. Because of its proven success in reducing thermally induced convection during melt growth of semiconductor crystals under terrestrial conditions, magnetic damping has been 
considered as a potential technique to eliminate the harmful g-jitter convection, or more correctly stated, to damp the convection beyond detection. Some basic studies have been carried out in this direction and numerical simulations have been conducted also for the melt systems where flow is primarily attributed mainly to thermal gradients $[8,9]$.

Thus far, very scarce work appears to have been done on the fundamental understanding of magnetic field effects on double diffusive convection in microgravity environment. The study of this type is of critical importance to space experiments involving binary alloy solidification. Some of these experiments are being planned for future Space Shuttle flights [7]. Similar experiments are being considered for the upcoming International Space Station. In this paper, we intend to develop a fundamental understanding of magnetic field effects on double diffusive convection induced by g-jitter in microgravity environment through extensive numerical simulations and analysis. For this purpose, a simple 2-D cavity geometry is considered and the Navier-Stokes equations, energy equations and solutal transport equations formulated within the framework of magnetohydrodynamics are solved using the finite element method. Numerical simulations are performed with the g-jitter data synthesized using Fourier series transformations and real g-jitter data taken directly from space flights, in combination with an external magnetic field. Computed results suggest that an external magnetic field can have a significant effect on double diffusive convection and a quiescent melt pool is possible with a strong field. The information and computational methodology should be useful for an understanding of physics governing the g-jitter induced double diffusive convection in a magnetic field and for designing and operating thermal fluids systems for space experiments involving electrically conducting binary melts.

\section{PROBLEM FORMULATION}

The model problem under consideration is schematically illustrated in Figure 2, where the coordinate system for analysis is also shown. A square cavity is filled with a binary liquid metal alloy melt. Its right side is fixed at a higher concentration $C_{H}$ and its left side a higher temperature $T_{H}$. One of the important characteristics is that the solutal Grashof number $\mathrm{Gr}_{\mathrm{S}}$ and the thermal Grashof number Grr to be approximately the same. The dimension of the square is 
set to $\mathrm{L}$, as nondimensionalized, equal to 1 . The upper and lower walls are kept adiabatic. The origin of the coordinate system is fixed at the lower left corner of the cavity. In microgravity, double diffusive melt convection arises because of the combined effect of the temperature and concentration gradients and the time varying gravity perturbation. The melt flow in turn will alter, in principle, both the thermal and concentration fields. A DC magnetic field is applied in the $\mathrm{x}$-direction. It interacts with the velocity field to produce an opposing Lorentz force to the local velocity, thereby resulting in the local velocity being reduced. Consequently, the convective transport of solutal elements is supressed.

In general, g-jitter is known to be random both in time and in direction (see Figure 1). However, for a certain period of time, g-jitter may be partially represented by a synthesized Fourier series via the fast Fourier transformation,

$$
\mathbf{g}^{*}\left(t^{*}\right)=\sum_{n=1}^{N} g_{n}^{*} \sin \left(2 \pi f_{n}^{*} l^{*}\right) \mathrm{e}
$$

where $g_{n}{ }^{*}$ and $f_{n}{ }^{*}$ are the amplitude and frequency of the nth acceleration component, respectively. Here the subscript * designates a dimensional quality.

The magntohydrodynamic form of the transport equations describing the g-jitter induced melt flow, heat and mass transport in the liquid pool system with an imposed magnetic field has here been established. These equations are written in a nondimensionalized forms as follows [10]:

$$
\begin{aligned}
& \nabla \cdot \mathbf{u}=0 \\
& \frac{\partial \mathbf{u}}{\partial t}+(\mathbf{u} \cdot \nabla) \mathbf{u}=-\nabla P+\nabla^{2} \mathbf{u}-\left(G r_{T} T+G r_{S} C\right) \mathbf{g}(\mathrm{t})+H a^{2}(\mathbf{u} \times \mathbf{b} \times \mathbf{b}) \\
& \frac{\partial T}{\partial t}+\mathbf{u} \cdot \nabla T=\frac{1}{P r} \nabla^{2} T \\
& \frac{\partial C}{\partial t}+\mathbf{u} \cdot \nabla C=\frac{1}{S c} \nabla^{2} C
\end{aligned}
$$


In the above equations, lengths are nondimensionalized by $L$, velocity by $v / L$, time by $L^{2} / v$, temperature by $\Delta T=T_{H}-T_{l}$, and concentration by $\Delta C=C_{H}-C_{L}$. Also use has been made of the Boussinesq's approximation, $\rho(T, C)=\rho_{0}\left(1-\beta_{T}\left(T-T_{0}\right)-\beta_{C}\left(C-C_{0}\right)\right)$, and $\mathbf{g}(\mathrm{t})$ is nondimensionalized by $\mathrm{g}_{0}=9.8 \mathrm{~m} / \mathrm{s}^{2}$.

The last term on the right hand side of Eq. (3) represents the Loretnz forces that result from the interaction of the applied magnetic field $\$$ and the local velocity. These forces always act in the opposite direction to the velocity and thus generate a local impedance to the flow. In writing Eq. (3) some simplifications regarding the behavior of the magnetic and electric fields have been incorporated. First, the effect of the magnetic field induced by the velocity is neglected, in comparison with the imposed magnetic field. This is permissible in that the magnetic Reynolds number is sufficiently small for the system being studied [11]. The theory of magnetohydrodynamics has further established that this condition holds true for almost all the laboratory experiments involving conducting fluids [10]. Also, with the magnetic field applied in the $x$ - and/or $y$-directions, the electric field (thus the electric potential) arising from the interaction of the magnetic field and the velocity can be shown to be zero and thus make no contribution to the Lorentz forces [12].

The above equations are subject to the physical constraints that are described by the following boundary conditions:

$$
\begin{aligned}
& u=0 \text { at } x=0 \& x=1 \text { and } y=0 \& y=1 \\
& \frac{\partial T}{\partial y}=\frac{\partial C}{\partial y}=0 \text { at } y=0 \& y=1 \\
& T=1, C=0 \text { at } x=0 \\
& T=0, C=1 \text { at } x=1
\end{aligned}
$$

\section{THE FINITE ELEMENT SOLUTION}

The above governing equations along with the boundary conditions are solved using the Galerkin finite element method. Following the procedures given in [13], the integral forms of the governing equations can be obtained with the result, 


$$
\begin{aligned}
& \left(\int_{\Omega} \psi \hat{j} \cdot \nabla \phi^{T} d V\right) \mathbf{U}_{j}=-\varepsilon\left(\int_{\Omega} \psi \psi^{T} d V\right) \mathbf{P} \\
& \left(\int_{\Omega} \phi \phi^{T} d V\right) \frac{d \mathbf{U}_{i}}{d t}+\left(\int_{2} \phi \mathbf{u} \cdot \nabla \phi^{T} d V\right) \mathbf{U}_{i}-\left(\int_{2} \hat{i} \cdot \nabla \phi \psi^{T} d V\right) \mathbf{P} \\
& +\left(\int_{\Omega} \nabla \phi \cdot \nabla \phi^{T} d V\right) \mathbf{U}_{i}+\left(\int_{\Omega}(\hat{i} \cdot \nabla \phi)\left(\hat{j} \cdot \nabla \phi^{T}\right) d V\right) \mathbf{U}_{j}+\left(\int_{\Omega} G r_{T} g_{i} \phi \theta^{T} d V\right) \mathbf{T} \\
& +\left(\int_{\Omega} G r_{S} g_{i} \phi \theta^{T} d V\right) \mathbf{C}=\int_{\partial \Omega} \mathbf{n} \cdot \tau \cdot \hat{i} \phi d S+\int_{\Omega} H a^{2}(\mathbf{u} \times \mathbf{b} \times \mathbf{b}) \phi d V \\
& \left(\int_{\Omega} \theta \theta^{T} d V\right) \frac{d \mathbf{T}}{d t}+\left(\int_{\Omega} \theta \mathbf{u} \cdot \nabla \theta^{T} d V\right) \mathbf{T}+\left(\int_{\Omega} \frac{1}{P r} \nabla \theta \cdot \nabla \theta^{T} d V\right) \mathbf{T}=-\int_{\sigma_{\Omega}} q_{T} \theta d S \\
& \left(\int_{\Omega} \theta \theta^{T} d V\right) \frac{d \mathbf{C}}{d t}+\left(\int_{\Omega} \theta \mathbf{u} \cdot \nabla \theta^{T} d V\right) \mathbf{C}+\left(\int_{\Omega} \frac{1}{S c} \nabla \theta \cdot \nabla \theta^{T} d V\right) \mathbf{C}=-\int_{\Omega \Omega} q_{c} \theta d S
\end{aligned}
$$

The integration defined in the above equations can be carried out numerically over each of discretized finite elements once the shape functions $\phi, \theta$, and $\psi$ are appropriately selected. The final results may be summarized in the following global matrix,

$$
\left[\begin{array}{ccc}
\mathbf{M} & 0 & 0 \\
0 & \mathbf{N}_{T} & 0 \\
0 & 0 & \mathbf{N}_{C}
\end{array}\right]\left[\begin{array}{c}
\dot{\mathbf{U}} \\
\dot{\mathbf{T}} \\
\dot{\mathbf{C}}
\end{array}\right]+\left[\begin{array}{ccc}
\mathbf{A}(\mathbf{U})+\mathbf{K}+\frac{1}{\varepsilon} \mathbf{E M}_{p}^{-1} \mathbf{E}^{T} & \mathbf{B}_{T} & \mathbf{B}_{C} \\
0 & \mathbf{D}_{T}(\mathbf{U})+\mathbf{L}_{T} & 0 \\
0 & 0 & \mathbf{D}_{C}(\mathbf{U})+\mathbf{L}_{C}
\end{array}\right]\left[\begin{array}{c}
\mathbf{U} \\
\mathbf{T} \\
\mathbf{C}
\end{array}\right]=\left[\begin{array}{c}
\mathbf{F} \\
\mathbf{G}_{T} \\
\mathbf{G}_{C}
\end{array}\right]
$$

where the coefficient matrices are computed by

$$
\begin{array}{ll}
\mathbf{M}_{p}=\int_{\Omega} \psi \psi^{T} d V^{\prime}: & \mathbf{N}_{T}=\int_{\Omega} \theta \theta^{T} d V \\
\mathbf{M}=\int_{\Omega} \phi \phi^{T} d V: & \mathbf{N}_{c}=\int_{\Omega} \theta \theta^{T} d V \\
\mathbf{E}_{j}=\int_{\Omega} \hat{j} \cdot \nabla \phi \psi^{r} d V & \mathbf{L}_{c}=\int_{2} \frac{1}{S c} \nabla \theta \cdot \nabla \theta^{T} d V \\
\mathbf{L}_{T}=\int_{\Omega} \frac{1}{P r} \nabla \theta \cdot \nabla \theta^{T} d V ; & \mathbf{A}(\mathbf{U})=\int_{2} \phi \mathbf{u} \cdot \nabla \theta^{T} d V
\end{array}
$$




$$
\begin{array}{ll}
\mathbf{D}_{C}(\mathbf{U})=\int_{2} \theta \mathbf{u} \cdot \nabla \theta^{T} d V ; & \mathbf{D}_{T}(\mathbf{U})=\int_{2} \theta \mathbf{u} \cdot \nabla \theta^{T} d V \\
\mathbf{B}_{T}=\int_{\Omega} G r_{T} \mathbf{g} \phi \theta^{T} d V & \mathbf{B}_{C}=\int_{\Omega} G r_{S} \mathbf{g} \phi \theta^{T} d V \\
\mathbf{G}_{T}=-\int_{\partial \Omega} 4 T \theta d S ; & \mathbf{G}_{C}=-\int_{\partial \Omega} q_{C} \theta d S \\
\mathbf{F}_{i}=\int_{\partial \Omega} \mathbf{n} \cdot \tau \cdot \hat{i} \phi d S & \\
\mathbf{K}_{i j}=\left(\int_{\Omega} \nabla \phi \cdot \nabla \phi^{T} d V\right) \delta_{i j}+\int_{\Omega}(\hat{i} \cdot \nabla \phi)\left(\hat{j} \cdot \nabla \phi^{T}\right) d V-\left(\int_{\Omega} b_{i} b_{j} \phi \phi^{T}\right) d V\left(1-\delta_{i j}\right) \\
+\left(\int_{\Omega} b_{2}{ }^{2} \delta_{1 i} \phi \phi^{T} d V\right) \delta_{i j}+\left(\int_{\Omega} b_{1}{ }^{2} \delta_{2 j} \phi \phi^{T} d V\right) \delta_{i j}
\end{array}
$$

It is noted that in cleveloping the above matrix equation, the pressure is approximated by the penalty method, and $\mathbf{P}$ in the momentum equation is substituted by $\frac{1}{\varepsilon} \mathbf{M}_{p}^{-1} \mathbf{E}^{T} \mathbf{U}$. The assembled global matrix equations are stored in the skyline form and solved using the Gaussian elimination method. The time integration employs the backward Euler (or implicit) time difference scheme, with automatic time step control [13].

\section{RESULTS AND DISCUSSION}

Extensive numerical simulations were performed using a finite element code that incorporates the transient finite element formulation described above. The code was developed by our research group over the past years and has been tested against many bench-mark problems and other available commercial thermal fluid flow computational packages. It has also been used to study the g-jitter induced double diffusive convective flows in the absence of a magnetic field [14]. As a result, only the magnetic field effect needs to be added to the code. To ensure its predictability of magnetic damping phenomena, the code is also tested against the analytical solution obtained for the thermally induced flows in a simple parallel plate configuration with a transverse magnetic field imposed [15]. There exists gratifying agreement between the numerical and analytical solutions as evidenced by the results appearing in Figure 3, thereby providing a validation of the finite element computational methodology presented.

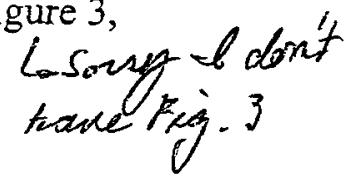

It is well known that a Hartmann boundary layer exists near the solid walls whenever an external magnetic field is imposed on a moving fluid, and for an oscillating flow the layer becomes even thimner [11]. This thin/ boundary layer can cause considerable numerical difficulties and introduce numerical errors if mesh is not carefully designed. Numerical tests 
were carried out to determine the grid dependence and computational accuracy the the used the worst scenario condition to be encountered in the study. The testing results suggest that about 3 to 5 nodes within the Hartmann boundary layer are required to ensure an accurate representation of the wall layer. Combined with the testing of mesh arrangement for computation of pure double diffusive convection [14], the final finite element mesh was chosen for the computation presented in this study. The mesh consists of 2259 -node elements with node spacing decreased from center towards the walls following an arithmetic progressive ratio of 0.3 .

For the results presented below, the parameters used for computations are: $\mathrm{Pr}=0.012$, $\mathrm{Gr}$

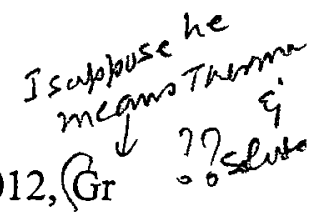
$=1.54 \times 10, \mathrm{Gr}=-1.54 \times 10 . \mathrm{Sc}=65.6$ and $\mathrm{Ha}=40 \sim 200$ unless otherwise indicated. These parameters were obtained based on a system being considered for a Space Shuttle flight [14]. The initial condition for the transient simulations is the steady+state fluid flow and concentration distribution in the same geometry. without an external magnetic field, which is induced by a microgravity field of $g=1 \times 10^{-6} g_{0}$, oriented in the y-direction. This would correspond to a typical quiet condition during a space flight. The flow, thermal and solutal distributions for the steady state condition were presented in the previous publication [14] and thus are omitted here. It is worth noting, though, that for the low Prandtl number fluids being considered here the thermal fields remain basically the same when the g-jitter perturbations become active. For this very reason, the thermal contours are not included in discussion. Also, the results discussed below are computed based on the situations in which the magnetic field is turned on when g-jitter sets in to investigate the magnetic damping effects. This is the optimal scenario from the standpoint of power consumption in space. Additional numerical simulations were also performed to investigate the cases where the magnetic field is on all the time including the quiet period and the results show that the magnetic damping effect is even more pronounced. Nonetheless, the results discussed below should serve well the objective of this paper, that is, to develop a fundamental understanding of He jitter induced double diffusive convection.

\section{A. Single frequency $g-j i t t e r$}


As stated earlier, g-jitter is random in nature and thus a single frequency representation is an oversimplified statement. However, the study of single frequency should be helpful for an understanding of the very basics of the flow behavior and hence the solute transport in the presence of an external magnetic field. Figure 4 contrasts the fluid flow and concentration distributions in the melt system with and without the presence of an external magnetic field (Ha $=120$, corresponding to $B=0.3$ Tesla) at a moment in time after a g-jitter component of $\mathrm{g}=1 \mathrm{x}$ $10^{-3} \mathrm{~g}_{0} \sin (0.2 \pi \mathrm{t})$ sets in. This type of disturbance is known to have a deleterious effect on melt flow and hence solutal transport in space. Apparently, even with a moderate magnetic field, the melt flow intensity is much reduced, which in turn makesthe concentration profile less distorted. Analysis of the additional results further illustrates that the period of time harmonic oscillation cycle remains the same as the driving g-jitter component, with or without the applied magnetic

- field. With an applied magnetic field, however, the phase angle is changed and is found to be a function of the magnitucle of the applied field, in addition to other known factors, such as, the location, the local velocity and the amplitude of the g-jitter component [14]. Also, the application of an external magnetic field results in a reduction of transient period over which the fluid flow evolves to a quasi-steady state time harmonic oscillation. For pure double diffusive convection, it was found that the subharmonic oscillation does not occur until the g-jitter perturbations become unrealistically high [14]. The applied magnetic field further delays the occurrence of sub-harmonic motions. Sub-harmonic motion comes from the nonlinear (or convective) term of the momentum equation. For the conditions being studied, the velocity gradient is smaller, although the main flow is strong, thereby causing the flow to oscillate with the same frequency as the driving force. Magnetic damping further reduces the magnitude of the velocity but the velocity gradient remains approximately the same level. As a result, subharmonic motion becomes even less likely with an applied field. Numerical simulations with diflerent magnetic field strengths also revealed that a stronger magnetic field produces a more pronounced damping effect and helps to further reduce the transient period and to make the final harmonic oscillation almost in phase with the driving g-jitter force.

Concentration in the system is strongly affected by the melt convection and the decrease of flow intensity should reduce the solutal striation in the melt pool. This is confirmed by the numerical results obtained for the above g-jitter condition with different magnetic fields. Figure 
5 depicts the transient development of the velocity components and concentrations at a few locations as a function of applied fields. Clearly, the use of magnetic fieldf produces strong damping effect on convection and thus smoothes out the solutal fluctuations. Also, a stronger magnetic field is more effective in suppressing the local velocity and hence the concentration non-uniformity.

\section{B. Multiple Frequency g-Jitter}

Multi-frequency g-jitter perturbations may be considered as a synthesized representation of g-jitter containing a range of frequencies and amplitudes (see Eq. (1)). They are useful for the study of the combined effects from various g-jitter components and their interaction with the applied magnetic fields. Numerical simulations were made with multiple g-jitter composed of 4 components with frequencies of $(1,0.1,0.01,0.001)$ and amplitudes of $\left(1.0 \times 10^{-3}, 1.0 \times 10^{-3}\right.$, $\left.1.0 \times 10^{-4}, 1.0 \times 10^{-5}\right)$, and with applied magnetic fields. Space experience showed that these $\mathrm{g}$ jitter components produce the most detrimental effects on the flow and hence solutal distributions in processing systems for space applications. From the fundamental point of view, it is also important to know what effects an applied magnetic field would have on the individual surle Fig. 6 g-jitter components when they all are present. The results obtained from the numerical model may are illustrated in Figures 6,7 and 8. Inspection of these results indicates that the applied

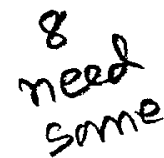
diffusive flows and hence are useful in reducing the solutal non-uniformity in the melt pool. discursim here.

Further numerical simulations revealed some additional interesting points that are unique to multiple frequency g-jitter with the presence of a magnetic field. First, the applied magnetic field is most effective in damping out the influence of the most detrimental g-jitter component, which is the component with the largest oscillation amplitude. Early studies [14] on g-jitter driven double diffusive convection in the absence of a magnetic field have concluded that for the conditions being studied the flows are essentially a superimposition of the flows by individual $g$ jitter components, and nonlinear effects such as subharmonic motions are a non-occurrence in space environment. Also, the component with the largest amplitude generates the most intensified convection. This flow field interacts with the applied magnetic field to give rise the strongest opposing Lorentz force. thereby producing the most pronounced damping effects. 
Second, with a large enough g-jitter, which is not realistic but theoretically possible, subharmonic motion becomes possible. Such a subharmonic motion can be suppressed by an appropriately chosen magnetic field and the nonlinear effect can be completely damped out. Moreover, a higher magnetic field produces a more effective damping result, again because of a larger Lorentz force. This is the same in the case of single frequency g-jitter, as discussed above. Finally, solutal non-uniformity decreases with the increasing magnetic field strength as a result of higher damping force. With a strong field, the local concentration oscillation may be suppressed beyond detection and a diffusion dominant growth condition is possible. The precise prediction of such conditions, of course, should have to consider the detailed crystal growth configurations and operating parameter, which is beyond the scope of the present paper, although the computer code is fully capable of doing so [16].

\section{Real g-Jitter}

In space environment. crew motions and other space related factors contributed to the departure from the perfect dynamic weightlessness condition or g-jitter perturbations. As discussed earlier, these perturbations are random in time and in direction (see Figure 1). Numerical simulations were performed for the real g-jitter data as given in Figure 1 in combination with an applied magnetic field. Some of the results are selectively presented in Figures 9,10 and 11 . As one might expect, no time harmonic motion is generated by such random g-jitter data. It is seen from these figures that an applied magnetic field is effective in reducing the double diffusive convection driven by these random g-jitter forces and hence the solutal striation. Several important points may be summarized below for these results. They are in principle consistent with the conclusions reached from the single- and multi-frequency studies described above. First, the recirculatingg flow pattern is unaltered, but the velocities are reduced with an applied magnctic field. Second, the magnetic field is more effective in damping out the large component effect or the g-jitter exhibiting the sudden spiking. This is clearly evident in Figure 10, where the largest percentage of velocity reduction is associated with the largest gjitter component. Third. the damping effects become more pronounced with the stronger magnetic fields. For the conditions studied, the concentration fluctuations caused by spiking in g-jitter decrease sharply with the increasing field strength, which is a direct consequence of a bigger reduction in velocity. In fact, with $\mathrm{Ha}_{2}=120$, the concentration irregularity caused by $\mathrm{g}-$ 


\section{$?$}

jitter spiking is almost completely smoothed out, as appears in Figure 11. Thus, for the melt system, an application of a magnetic field measured by $\mathrm{Ha}=120$ should suppress the melt convection nough so as to make the solutal transport process a diffusion dominant event.

\section{CONCULDING REMARKS}

This paper has presented a numerical study of the g-jitter driven double diffusive convective flow, thermal and concentration distributions in binary alloy melt systems subject to an external magnetic field. The study is based on the finite element solution of the transient magnetohydrodynamic equations governing the momentum, thermal and solutal transport in the melt pool. Extensive numerical simulations were performed using both the synthesized singleand multi-frequency g-jitter as well as the real g-jitter data taken during space flights with or without an applied magnetic field. Computed results indicate that the main melt flow is in essence a combination of velocity components driven by each individual g-jitter components, whether or not an external magnetic field is present. The flow field is characterized by a recirculating convection loop, which is caused by both thermal and solutal gradients and oscillates in time with a defined frequency equal to that of the driving g-jitter force. An applied magnetic field does not alter the recirculating pattern and for the oscillation period, but it changes the phase angle. More important, it interacts with the flow field to produce an opposing Lorentz force that in turn suppresses the existing double diffusive convection. As a result, the solutal striation and time fluctuations in the melt are reduced. Also, the transient period over which the velocity reaches a quasi-steady state time harmonic oscillation is shorter with an applied field than with it. The transient period for the concentration field, however, is much longer than that for the velocity, and is practically independent of an applied magnetic field. It is governed by solutal diffusion, and can be as long as 500 periods of oscillation before the concentration field finally reaches a quasi-steady state time harmonic oscillation. For a given field strength, the magnetic damping effect is more pronounced on the velocity associated with the largest g-jitter component present and/or the g-jitter spiking peaks. Furthermore, damping effects (i.e., the reduction of both flow intensity and concentration non-uniformity and fluctuations) become more pronounced with increasing magnetic field strength $\not$. A higher magnetic field is more helpful in bringing the convection oscillation in phase with the g-jitter driving force. Numerical results further indicate that with appropriately selected magnetic field 
strength the convective flows caused by g-jitter can be suppressed sufficiently so that the solutal transport in the melt is dominated by molecular diffusion.

\section{ACKNOWLEDGMENT}

The authors gratefully acknowledge the support of this work by NASA (Grant \#: NCC8-92). 


\section{REFERENCES}

1. H. C. de Groh III and E. S. Nelson, On Residual Acceleration During Space Experiments. ASME Winter Annual Meeting. Chicago, Nov 6-11, HTD-Vol 290, pp23-33, 1994.

2. S. Maruyama, T. Shibata, K. Tsukamoto, and K. Shimizu, Measurement of Solutal and Thermal Diffusion in Systems Subjected to Rapid Cooling under Microgravity during Parabilic Flight, Heat Transfer - Japanese Research, vol. 27, no. 2, pp. 114-129, Scripta Technica Inc., 1998.

3. R. Monti, and R. Savino, G-sensitivity of Microgravity Experimentation - Fundamental of Disturbance Response, Microgravity Science and Technology, Vol. 11, no. 2, pp. 53-58, 1998.

4. J. I. D. Alexander, and R.M. Banish, Modeling G-sensitivity of Low-gravity Experiments, Microgravity Science and Technology, vol. 11, no 3, pp. 90-95, Carl Hanser Verlag, 1998.

5. M.J.B. Rogers and J.I.D. Alexander, Analysis of Spacelab 3 Residual Acceleration Data, J. Spacecraft and Rockets, vol. 28, no.6, pp 707-712, 1991.

6. J. I. Ramos, G-jitter Effect on Mass Transfer in Annular Liquid Jets, Int. J. Numerical Methods for Heat and Fluid Flow, vol. 6, no. 5, pp. 17-28, 1996.

7. A. V. Bune, D. C. Gillies, and S. L. Lehoczky, Effect of Gravity on the Double Diffusive Convection during Directional Solidification of a Non-dilute Alloy with Application to $\mathrm{HgCdTe}$, Proc. of SPIE, The International Society for Optical Engineering, vol. 3792, Jul.19Jul.21, pp. 177-182, 1999.

8. J. Baumgartl and G. Muller. J. C.rystal Growth Vol. 169, 582-586, 1996.

9. N. Ma and J. S. Walker, Magnetic damping of buoyant convection during semiconductor crystal growth in microgravity: Spikes on residual acceleration. Physics of fluids. 8(4), 944, 1996.

10. S. Chandrasekhar, Hydrodynamic and Hydromagnetic Stability. Dover, New York, 1981.

11. Li, B. Q., G-jitter induced flows in a transverse magnetic field. Int. J. Heat \& Mass Trans., Vo. 39, No. 14, pp. 2853-2860, 1996.

12. G. M. Opeper and J. Szekely. The effect of an externally imposed magnetic field on buoyancy driven flow in a rectangular cavity. J. Crystal Growth vol 64, 505-515, 1983.

13. S. P. Song and B. Q. Li, Surface deformation and Marangoni Flow in Electrostatically levitated droplets, Int. J. Heat Mass Transf., Vol. 43, 3589-3606, 2000.

14. Shu, Y., Li, B. Q. and de Groh, H. C. Numerical study of g-jitter induced double diffusive convection. Numerical Heat Transfer, to appear. 
15. Pan, B. and Li, B. Q. Effect of magnetic fields on oscillating mixed convection. Int. J. Heat \& Mass Transfer, Vol. 41, No. 17, 2705-2710, 1998.

16. Rule, T., Li, B. Q. and Lynn, K., Finite element modeling of thermal and fluid flow phenomena in melt growth of CdTe single crystals. In the Proceedings of the ASME Heat Transfer Division, ASME, Orlando, FL, Nov. 2000. 


\section{Figure Captions}

Figure 1. Signature of $g$-jitter data in the $x$ and $y$ directions a function of time registered by an accelerometer aboard Space Shuttle, where time is measured in seconds.

Figure 2. Schematic representation of g-jitter driven oscillating convection in a 2-D cavity in the presence of a magnetic field.

Figure 3. Comparison of finite element solution and analytical solution for a single frequency gjitter induced thermal convection in a parallel plate channel subjected to a transverse magnetic field.

Figure 4. The velocity and concentration field induced by a single frequency g-jitter driving force in the $y$-direction with and without an external magnetic field. (a) Velocity ( $t=14.33163$, $\mathrm{Ha}=0, \mathrm{U}_{\max }=0.6367$ ), (b) Concentration $(\mathrm{t}=14.33163, \mathrm{Ha}=0)$, (c) Velocity $(\mathrm{t}=14.33163, \mathrm{Ha}=120$, $\left.\mathrm{U}_{\max }=0.1558\right)$ and $(\mathrm{d})$ Concentration $(\mathrm{t}=14.33163, \mathrm{Ha}=120)$.

Figure 5. Effects of applied magnetic fields on the time variation of velocity component $\mathrm{U}, \mathrm{V}$ and concentration with oscillating gravity. $(a, c, e)$ at $(0.5,0.9220)$ and $(b, d, f)$ at $(0.9220,0.5)$.

Figure 6. The velocity and concentration field under multiple frequency driven force and with and without magnetic field. (a) Velocity ( $\mathrm{t}=2.688364, \mathrm{Ha}=0, \mathrm{U}_{\max }=3.363$ ), (b) Concentration $(\mathrm{t}=2.688364, \mathrm{Ha}=0)$, (c) Velocity $\left(\mathrm{t}=2.688364, \mathrm{Ha}=40, \mathrm{U}_{\max }=0.4488\right)$ and (d) Concentration $(\mathrm{t}=2.688364, \mathrm{Ha}=40)$.

Figure 7. The dynanic development of velocity and concentration distribution with multiple oscillating gravity with and without magnetic field at $(0.9220,0.5)$ : (a, c, e) at $\mathrm{Ha}=0$ and (b, d, f) at $\mathrm{Ha}=120$.

Figure 8. Evolution of concentration as affected by the presence of magnetic field at $(0.5$, 0.9220). (a) $\mathrm{Ha}=0$ : (b) $\mathrm{Ha}=40$ : (c) $\mathrm{Ha}=120$ and (d) $\mathrm{Ha}=200$.

Figure 9. Magnetic clamping effects on the double diffusive convection and solutal distribution induced by real g-jitter driven force with and without magnetic field. (a) Velocity ( $t=0.2698170$, $\left.\mathrm{Ha}=0, \mathrm{U}_{\max }=0.1579\right)$, (b) Concentration $(\mathrm{t}=0.2698170, \mathrm{Ha}=0)$, (c) Velocity $(\mathrm{t}=0.269817$, $\left.\mathrm{Ha}=200, \mathrm{U}_{\max }=0.2957 \times 10^{-1}\right)$ and (d) Concentration $(t=0.269817, \mathrm{Ha}=200)$.

Figure 10. Time variation of velocity component $U$ and $V$ under real g-jitter and with and without magnetic field. (a. c) at $(0.9220,0.5)$ and (b. d) at $(0.5,0.9220)$.

Figure 11. Time variation of concentration distribution under real g-jitter and with and without magnetic field. (a) concentration at $(0.9220,0.5)$ and (b) concentration at $(0.5,0.9220)$. 

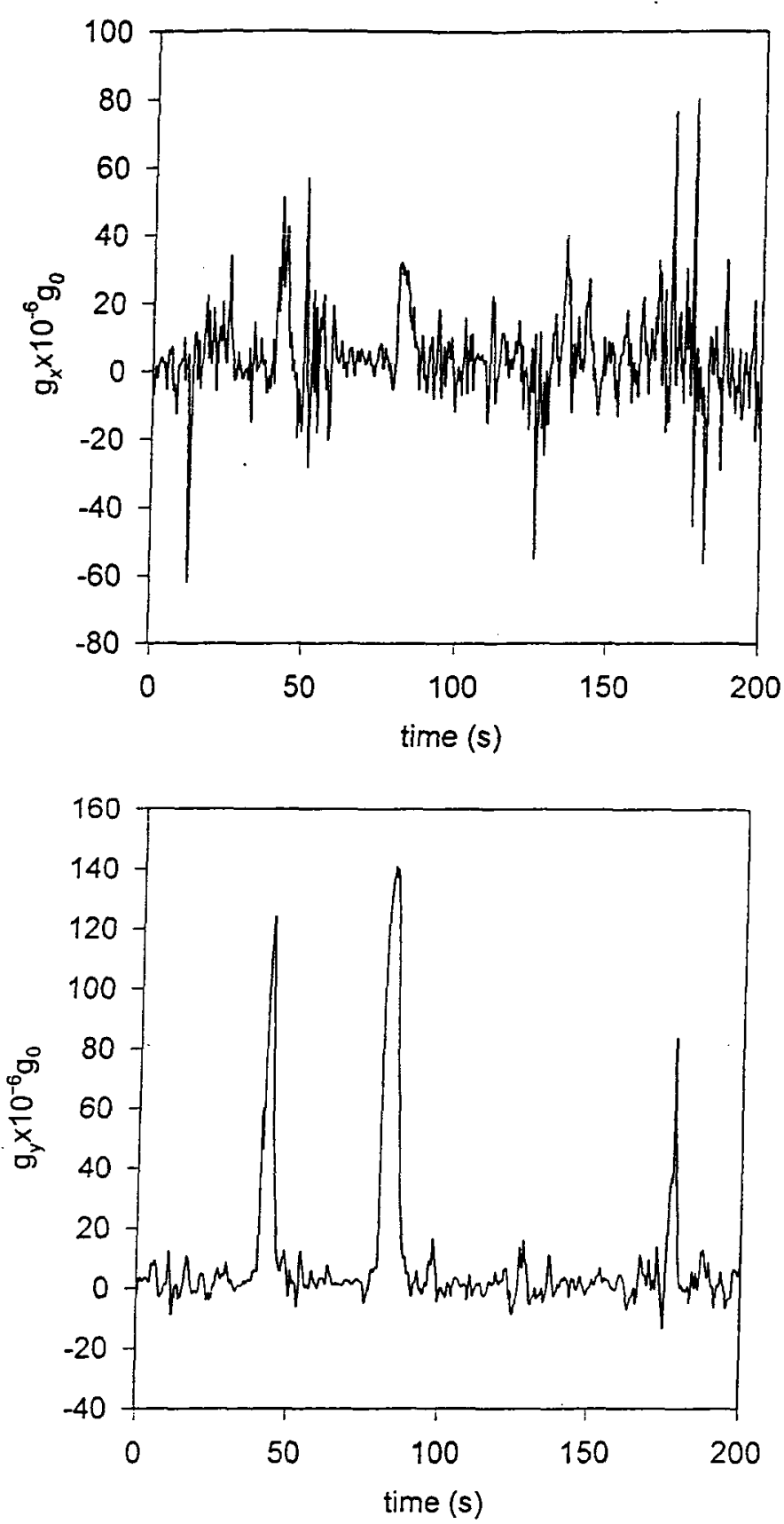

Figure 1. Signature of $\mathrm{g}$-jitter data in the $\mathrm{x}$ and $\mathrm{y}$ directions as a function of time registered by an accelerometer aboard Space Shuttle, where time is measured in seconds. 


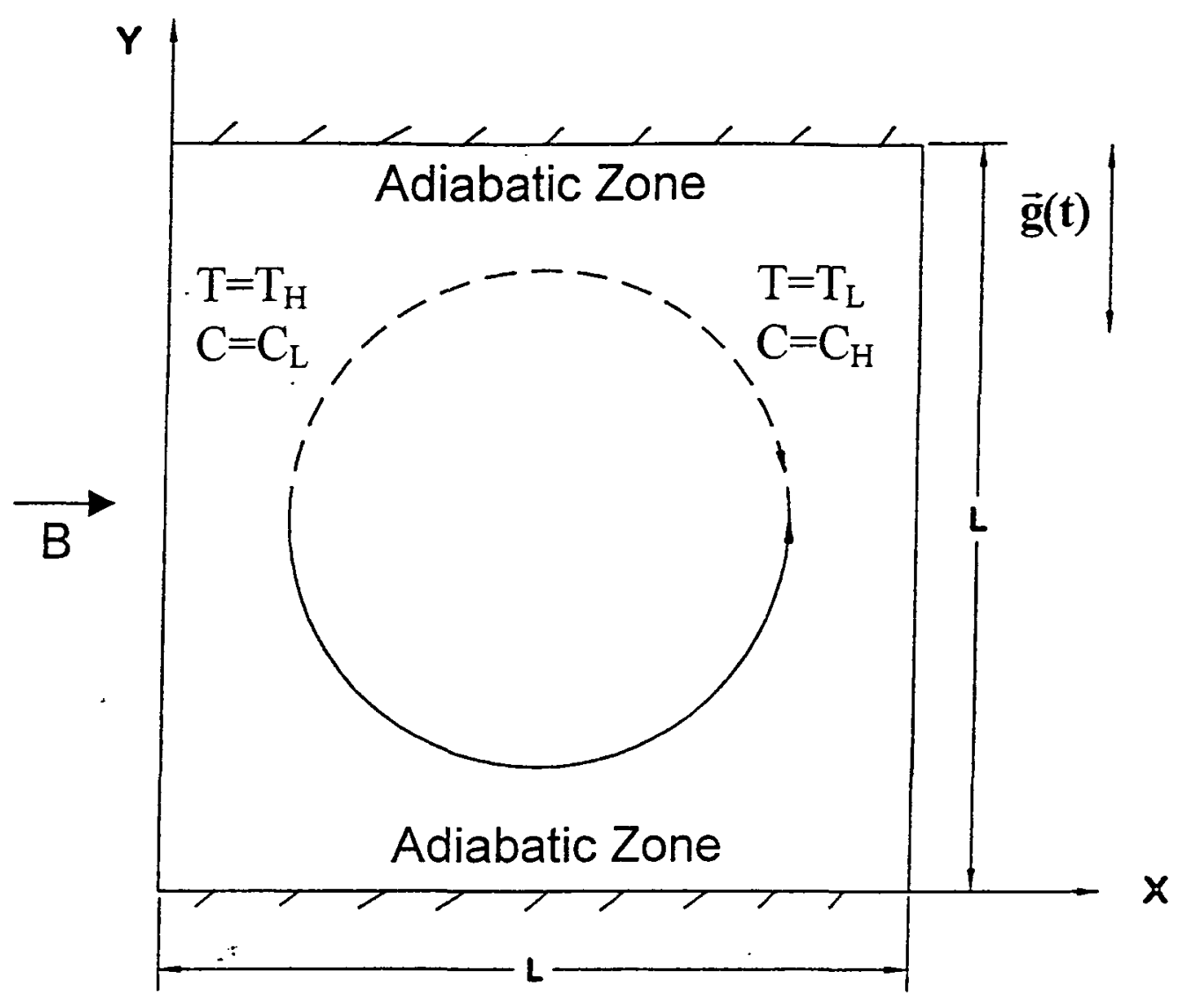

Figure 2. Schematic representation of g-jitter driven oscillating convection with magnetic field in a 2-D cavity in the presence of a magnetic field under microgravity condition. 


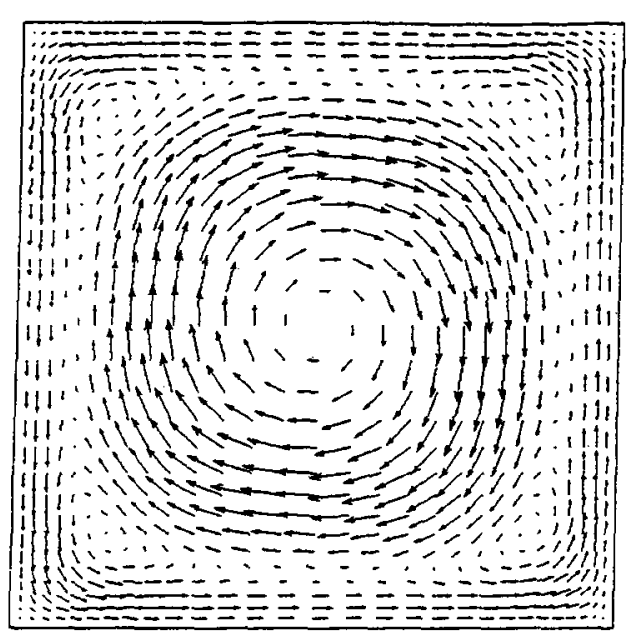

(a)

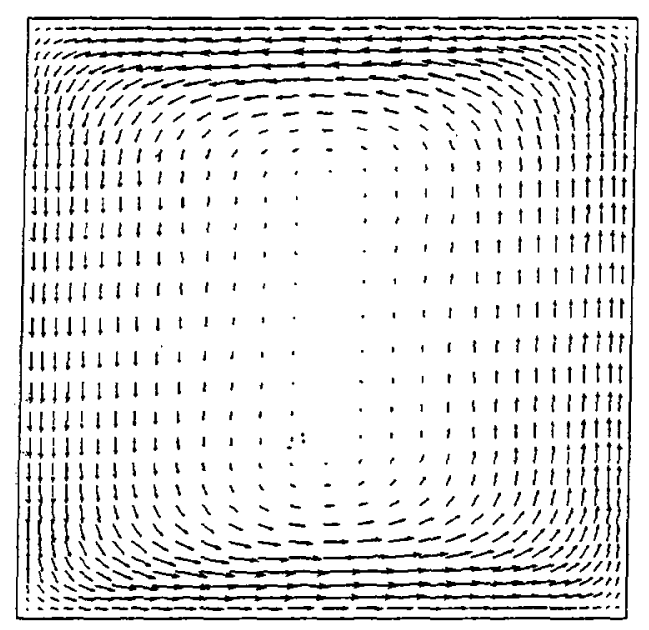

(c)

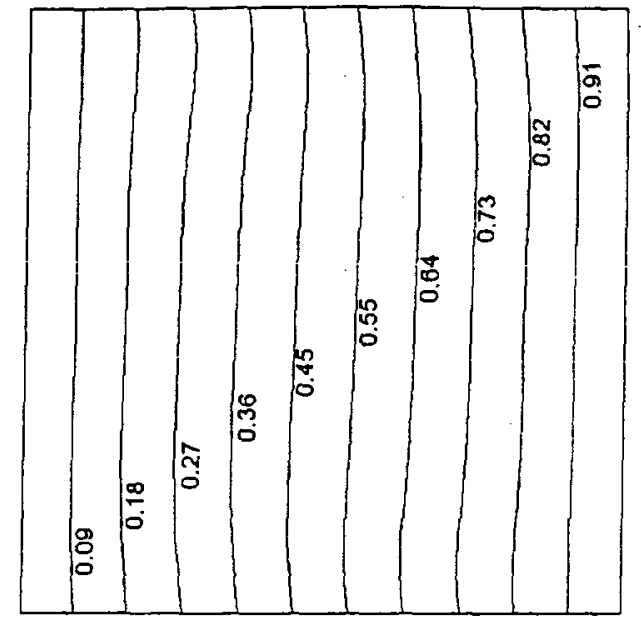

(b)

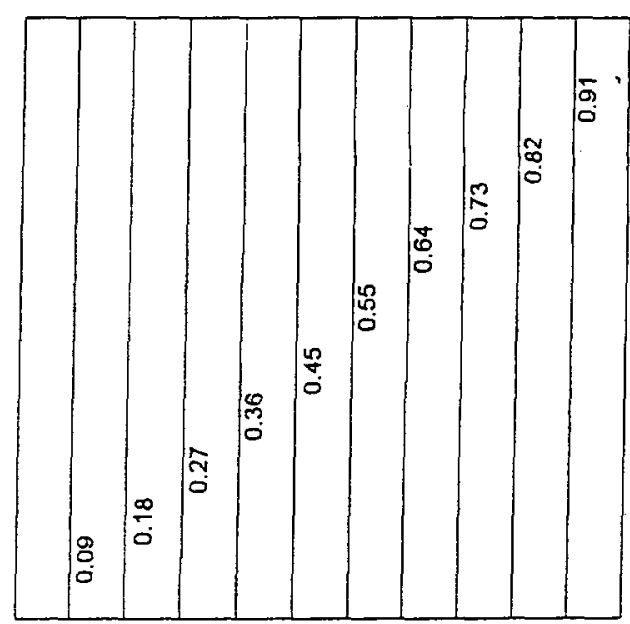

(d)

Figure 4. The velocity and concentration field induced by a single g-jitter driven force in y direction with and without an external magnetic field. (a) Velocity $(t=14.33163, \mathrm{Ha}=0$, $\left.\mathrm{U}_{\max }=0.6367\right)$, (b) Concentration ( $\left.t=14.33163, \mathrm{Ha}=0\right)$, (c) Velocity $(\mathrm{t}=14.33163, \mathrm{Ha}=120$, $\left.\mathrm{U}_{\max }=0.1558\right)$ and (d) Concentration ( $\left.\mathrm{t}=14.33163, \mathrm{Ha}=120\right)$. 

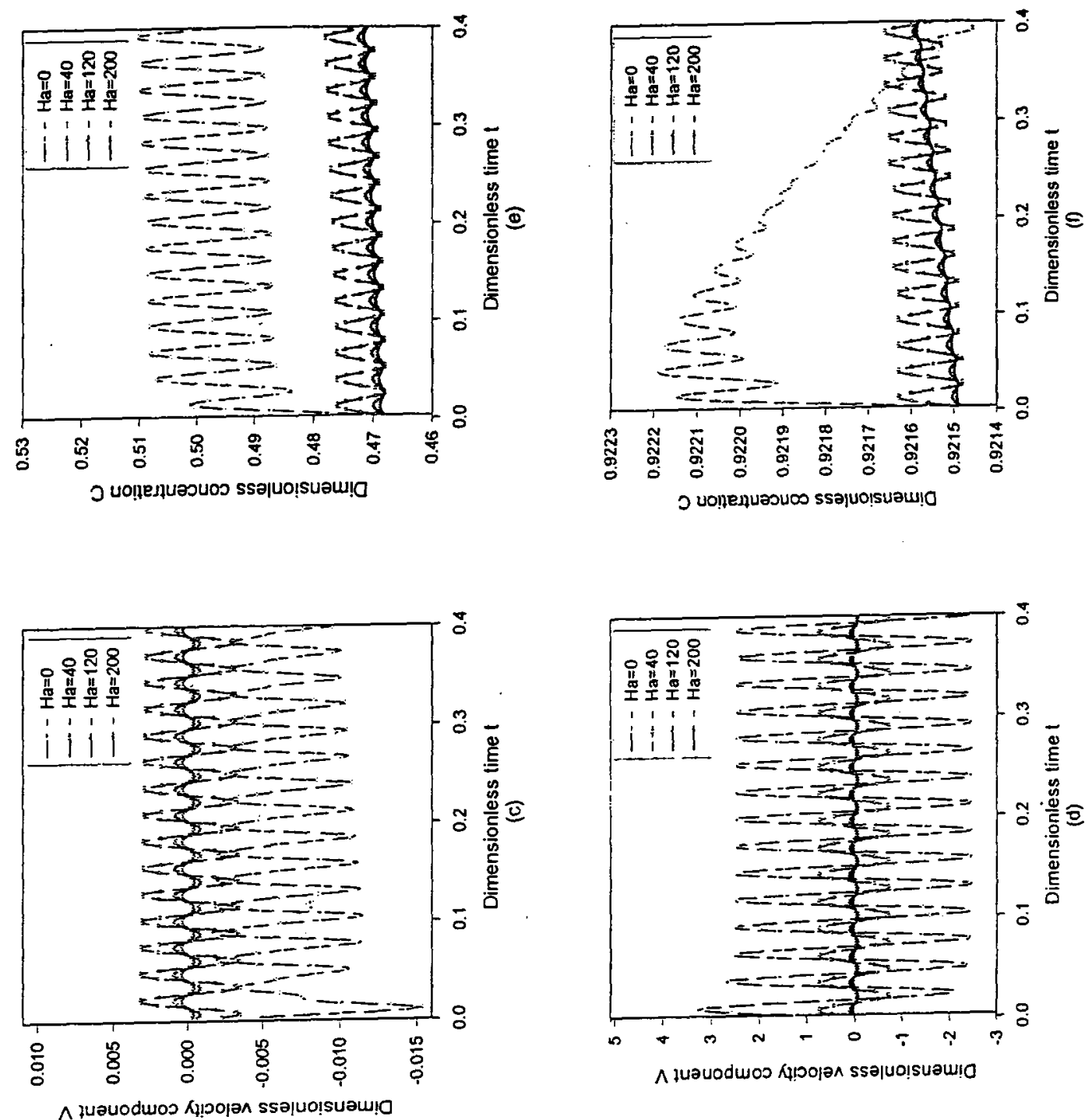

$\wedge$ juauoduos kịpojan sseluo! suaulo
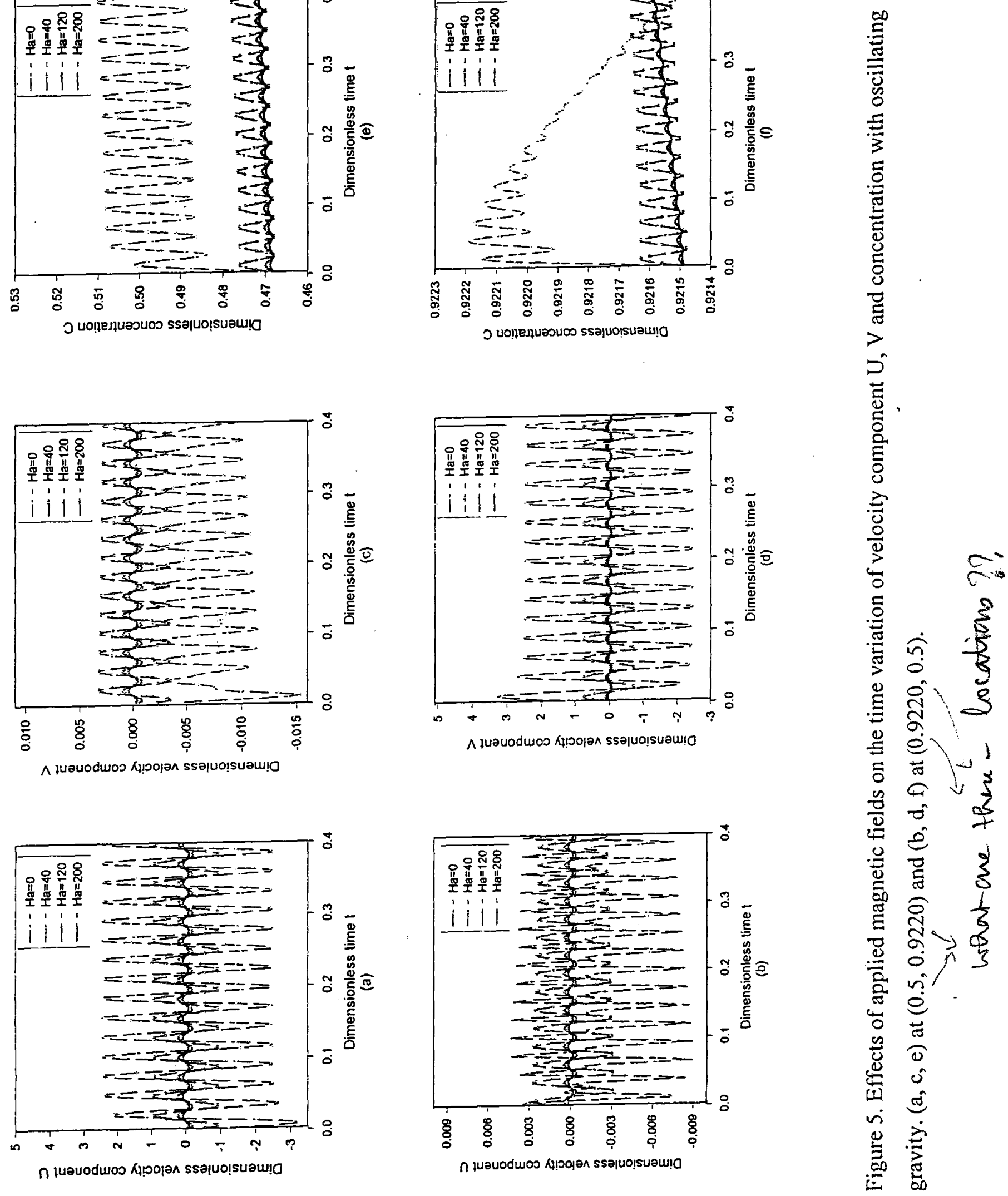


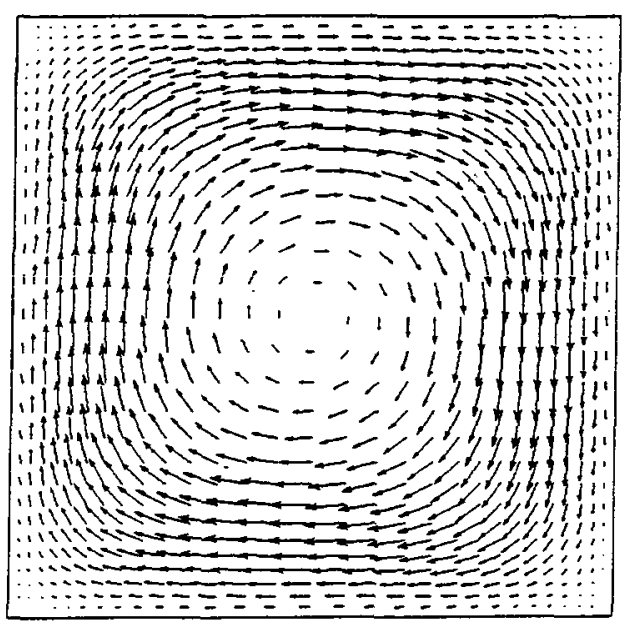

(a)

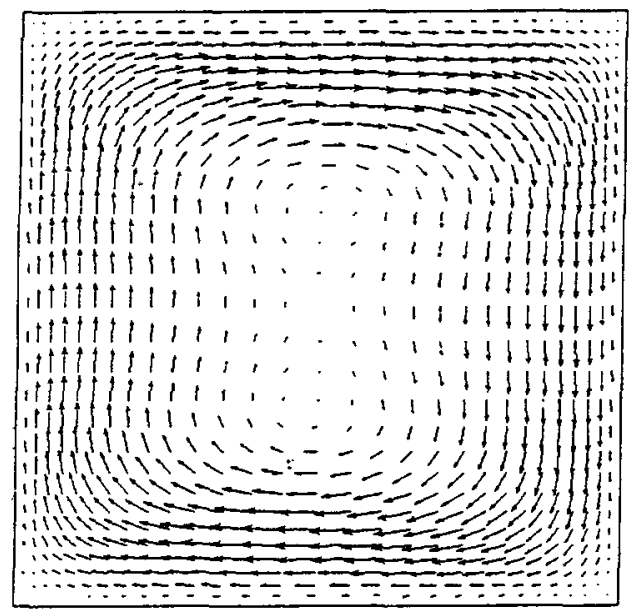

(c)

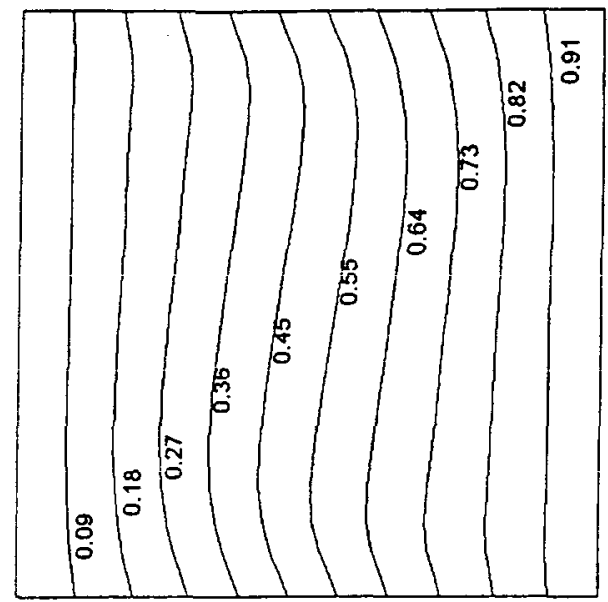

(b)

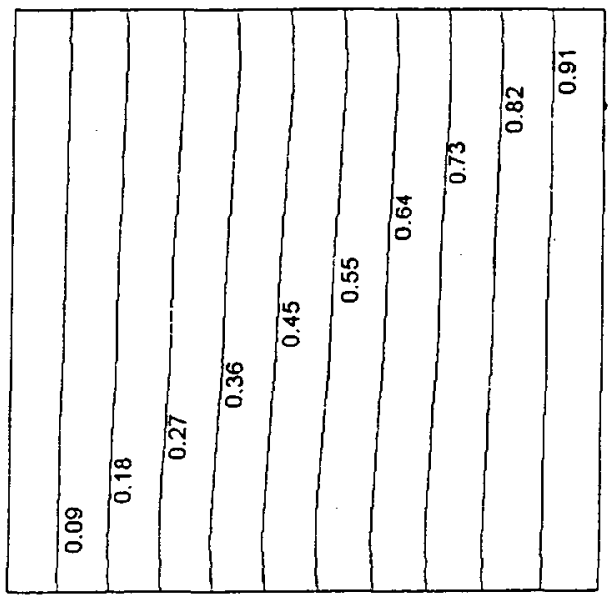

(d)

Figure 6. The velocity and concentration field under multiple frequency driven force and with and without magnetic field. (a) Velocity $\left(t=2.688364, \mathrm{Ha}=0, U_{\max }=3.363\right)$, (b) Concentration ( $t=2.688364, \mathrm{Ha}=0)$, (c) Velocity $\left(\mathrm{t}=2.688364, \mathrm{Ha}=40, \mathrm{U}_{\max }=0.4488\right)$ and (d) Concentration ( $\mathrm{t}=2.688364, \mathrm{Ha}=40)$. 

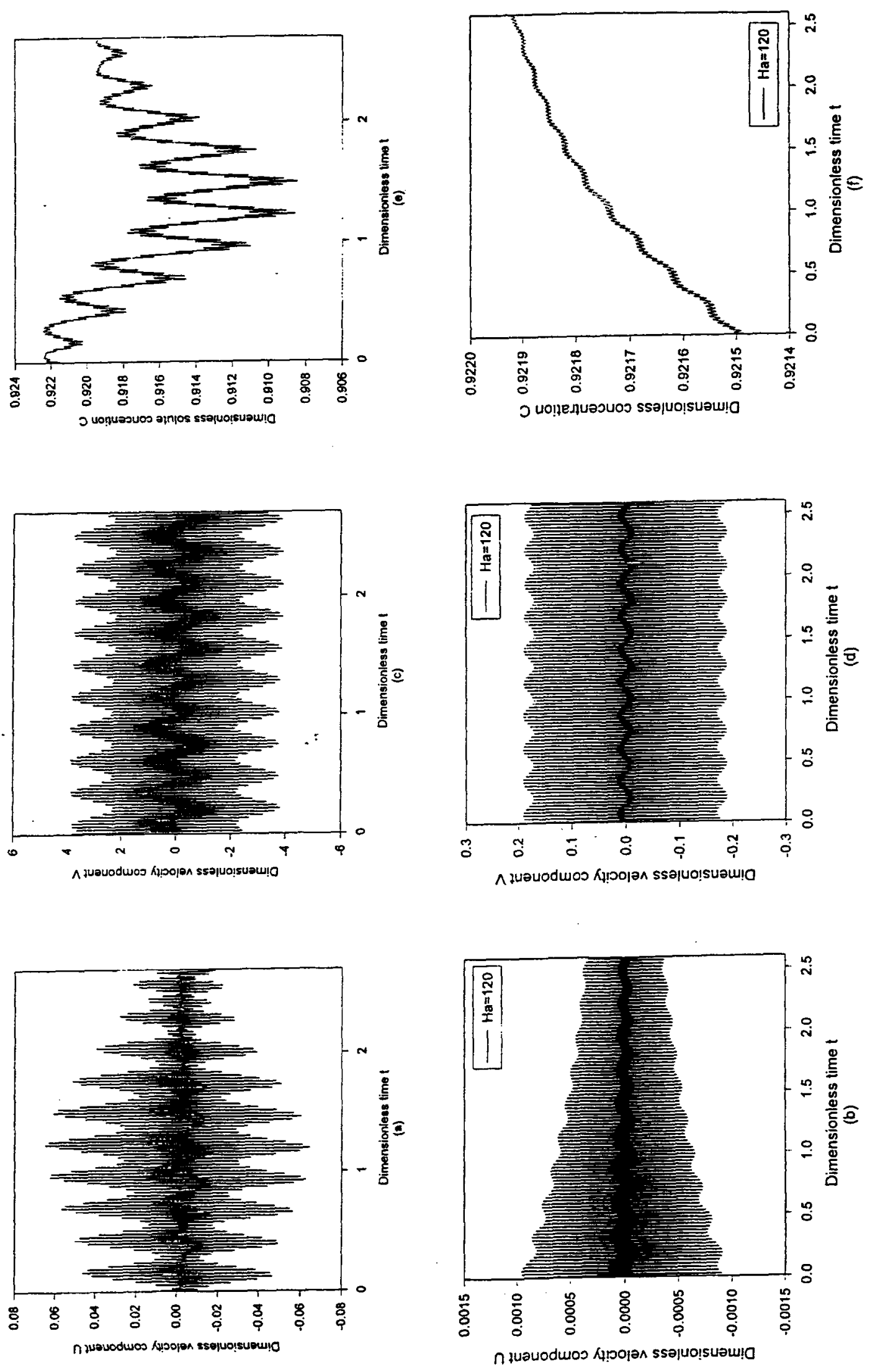

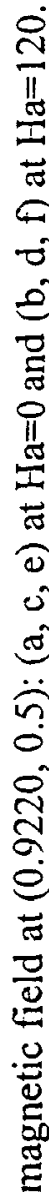




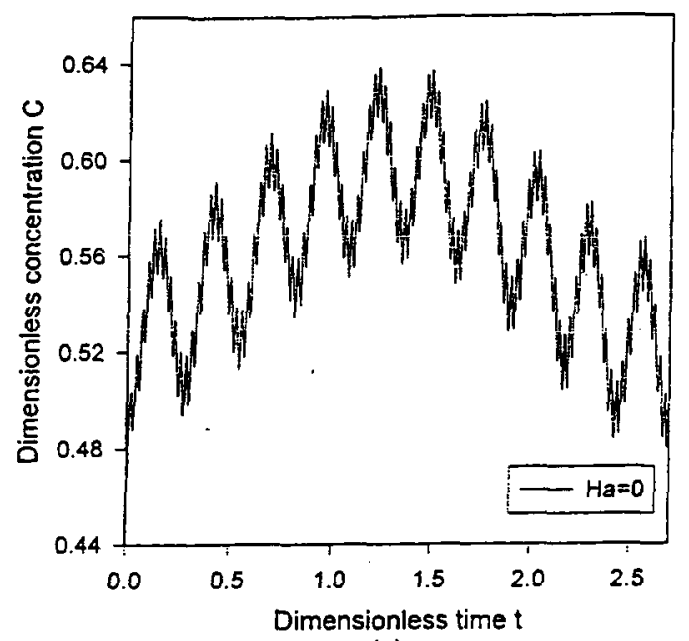

(a)

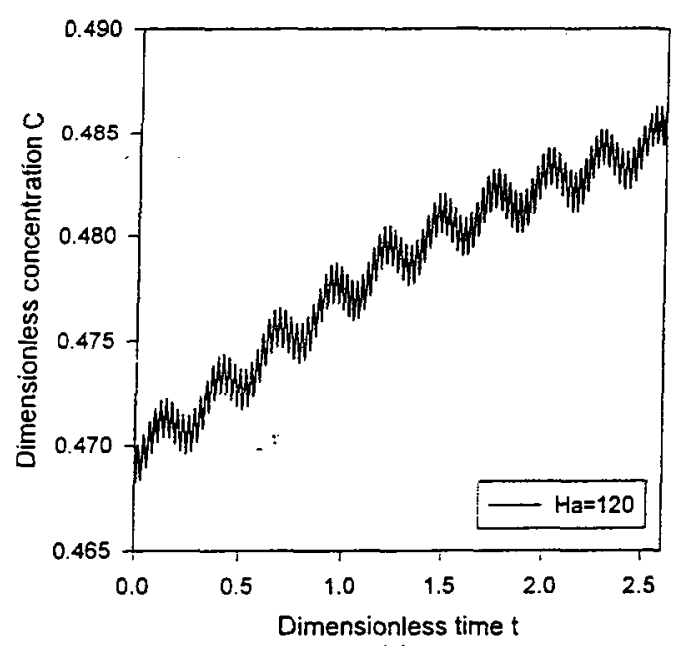

(c)

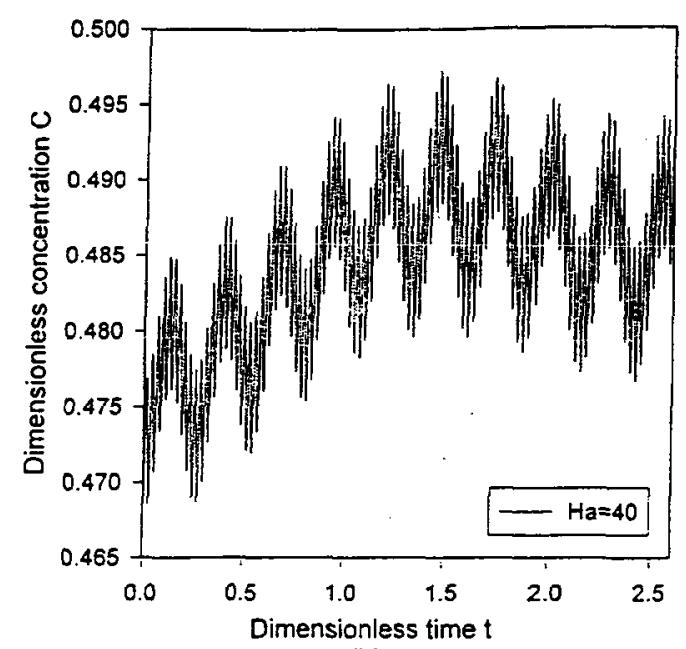

(b)

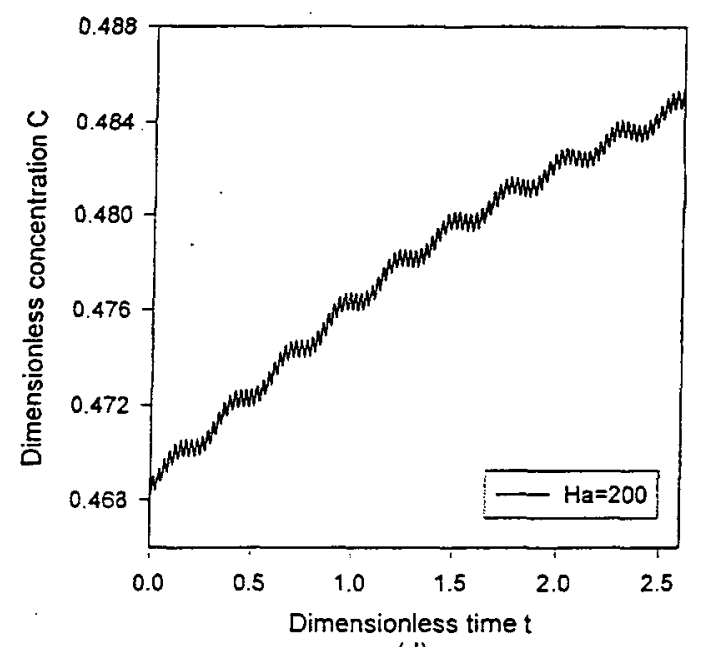

(d)

Figure 8. Evolution of concentration as affected by the presence of magnetic field at (0.5, 0.9220). (a) $\mathrm{Ha}=0$; (b) $\mathrm{Ha}=40$; (c) $\mathrm{Ha}=120$ and (d) $\mathrm{Ha}=200$. 


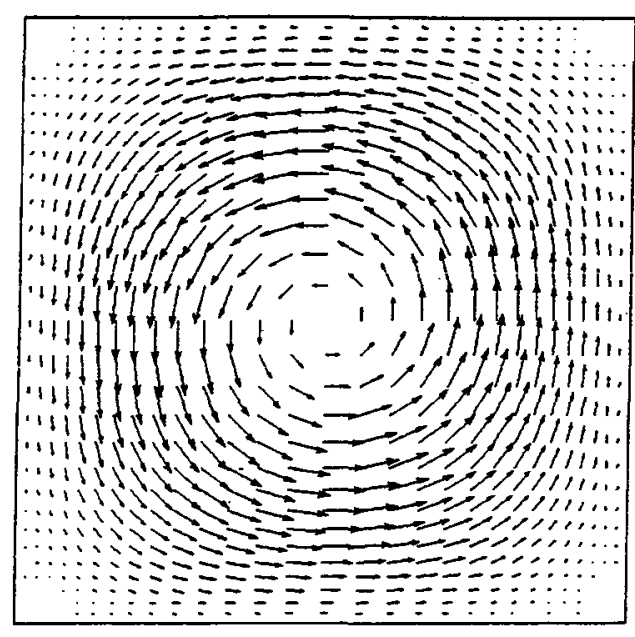

(a)

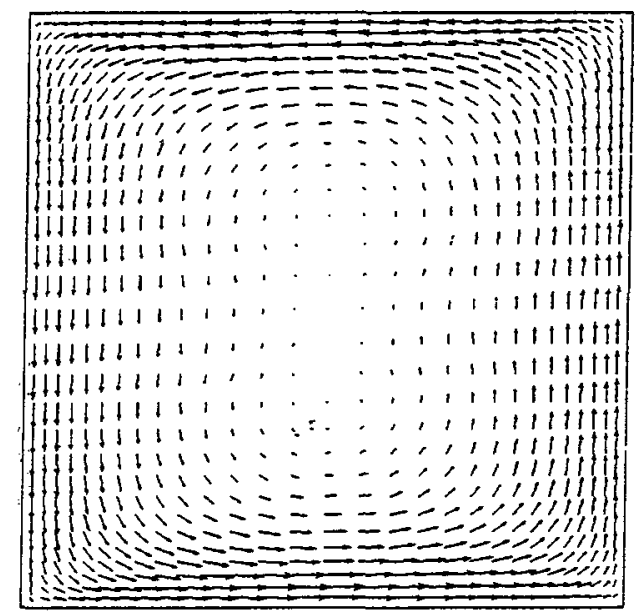

(c)

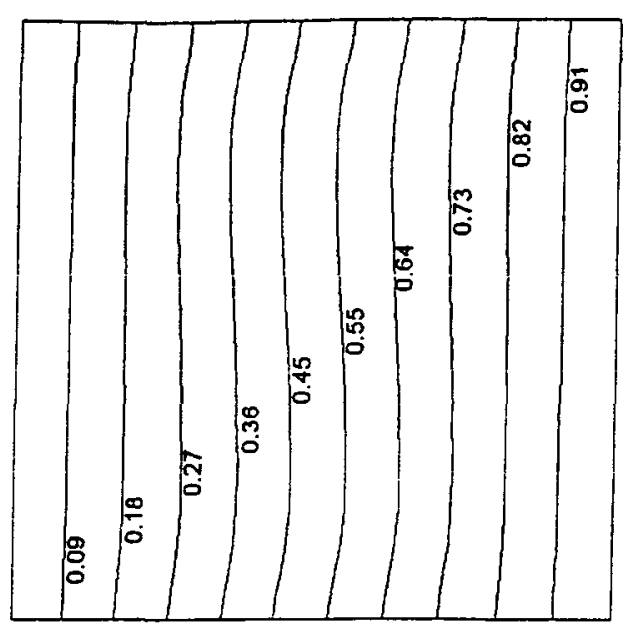

(b)

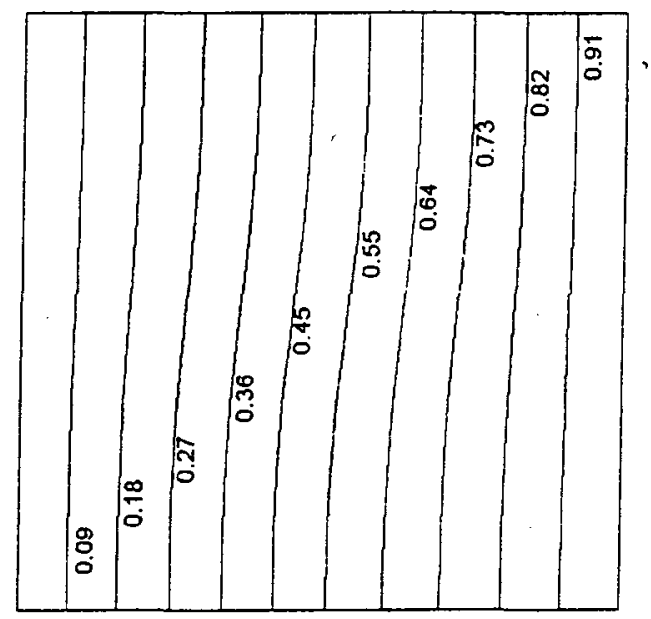

(d)

Figure 9. Magnetic damping effects on the double diffusive convection and solutal distribution induced by real g-jitter driven force with and without magnetic field. (a) Velocity ( $t=0.2698170, \mathrm{Ha}=0, U_{\max }=0.1579$ ), (b) Concentration ( $\left.t=0.2698170, \mathrm{Ha}=0\right)$, (c) Velocity $\left(t=0.269817, \mathrm{Ha}=200, U_{\max }=0.2957 \times 10^{-4}\right)$ and (d) Concentration $(t=0.269817$, $\mathrm{Ha}=200$ ). 